\title{
Salicylic acid-induced photosynthetic adaptability of Zea mays L. to polyethylene glycol-simulated water deficit is associated with nitric oxide signaling
}

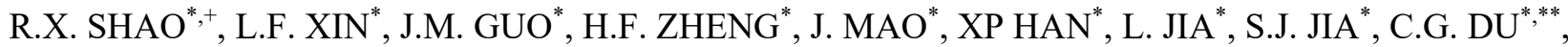 \\ R. SONG ${ }^{*,+}$, Q.H. YANG ${ }^{*,+}$, and R.W. ELMORE ${ }^{* * *}$ \\ Collaborative Innovation Center of Henan Grain Crops and State Key Laboratory of Wheat and Maize Crop \\ Science/College of Agronomy, Henan Agricultural University, Zhengzhou, 450 005, China* \\ Department of Biology, Montclair State University, Montclair, NJ 07043, USA** \\ Department of Agronomy and Horticulture, University of Nebraska-Lincoln, Lincoln 68583, USA ${ }^{* * *}$
}

\begin{abstract}
Salicylic acid (SA) and nitric oxide (NO) form a new group of plant growth substances that cooperatively interact to promote plant growth and productivity. Water deficit (WD) stress is a major limiting factor for photosynthesis, which in turn limits crop yield. However, the mechanism of SA and NO in stimulating photosynthesis has not yet been elucidated. Therefore, in this study, we investigated the SA- and NO-mediated photosynthetic adaptability of maize seedlings to WD in terms of photosynthetic parameters, activities and mRNA levels of $\mathrm{CO}_{2}$ assimilation enzymes. Our results showed that SA alleviated the WD-induced reduction of photosynthetic performance. The activities of Rubisco and Rubisco activase enzymes increased significantly due to SA pretreatment. Moreover, higher transcription rates of $R b c \mathrm{~L}, Z m R C A \alpha$ and $Z m R C A \beta$ mRNA further confirmed the effects of SA on $\mathrm{CO}_{2}$ assimilation. WD or SA-induced decreases or increases of $\mathrm{CO}_{2}$ assimilation ability were further decreased after c-PTIO addition.
\end{abstract}

Additional key words: chlorophyll fluorescence transients; gene expression; nitric oxide scavenger; photosynthetic characteristics.

\section{Introduction}

Maize (Zea mays L.) is one of the most important food crops in China and ranks third worldwide. Maize suffers from biotic and abiotic stress at different growth stages. Water deficit (WD) is one of limiting factors that adversely affect plant growth, development, survival, and crop productivity (Serraj et al. 2011). Maize is also considered one of the most WD-sensitive crops. An estimated 15$20 \%$ of the grain yield of maize is lost each year due to $\mathrm{WD}$, and such losses may increase further as WD become more frequent and severe with global climate change (FAOSTAT 2010). WD elicits a series of physiological responses including early reduced stomatal conductance $\left(g_{\mathrm{s}}\right)$ and photochemical inhibition, and a reduction in photosynthesis (Sikder et al. 2015).
Nitric oxide (NO), a gaseous free radical, is a known mediator of many physiological functions in animals. The identification of biological targets and functions of NO in mammals led to the investigation of its role in the physiological processes of plants (Hancock 2012). This led to the finding that plants can produce NO. NO is considered an essential signaling molecule in a wide range of physiological processes, such as seed germination, root organogenesis, stomatal movement, chlorophyll (Chl) biosynthesis, flowering, and senescence (Corpas et al. 2011, Liao et al. 2012, Tossi et al. 2012, Procházková et al. 2013). However, some studies also implicate NO as a stress-inducing agent in plants (Bajguz 2014).

Received 23 May 2017, accepted 14 February 2018, published as online-first 12 July 2018.

${ }^{+}$Corresponding authors; phone: +86-371-56990239 (R.X. Shao), e-mail: shaoruixin@henau.edu.cn; yangqinghua@henau.edu.cn Abbreviations: cGMP - cyclic guanosine monophosphate; Chl - chlorophyll; c-PTIO - 2-4-carboxyphenyl-4,4,5,5-tetramethylimidazoline-1-oxyl-3-oxide; $C_{\mathrm{i}}$ - intercellular $\mathrm{CO}_{2}$ concentration; $\mathrm{F}_{\mathrm{v}} / \mathrm{F}_{\mathrm{m}}$ - maximum photochemical efficiency of PSII; $g_{\mathrm{s}}-$ stomatal conductance; LA - leaf area; MAPK - mitogen-activated kinases; NO - nitric oxide; PEG - polyethylene glycol; $\mathrm{PI}_{\mathrm{ABS}}$ - photosynthesis performance in PSII electron transport; $P_{\mathrm{N}}$ - photosynthetic assimilation rate; RCA - Rubisco activase; RCs - per active reaction centers; RWC - relative water content; SA - salicylic acid; WD - water deficit; $W_{U E}$ - intrinsic water-use efficiency.

Acknowledgements: This research was financially supported by Open Foundation of CAAS/Key Laboratory of Crop Water Use and Regulation, Ministry of Agriculture (FIRI2016-01-02), Key Technology Developing Program of Henan Province (152102110069), and National Agricultural Science and Technology Achievement Transformation Project (2014D00000081). 
Under WD conditions, chloroplasts become one of the endogenous NO cellular sources; it has been assumed that there are binding sites for NO within PSII between the primary and secondary quinone acceptors (Jasid et al. 2006). NO protects the critical functional proteins in the PSII complex during drought stress indicating its regulatory role in photosynthesis. For example, in Triticum aestivum L, NO restricted the WD-induced reduction in transcription of Psb A gene that encodes the D1 protein of the PSII complex (Wang et al. 2011, Procházková et al. 2013). Additionally, exogenous NO ameliorates WD stress-induced inhibitory growth by enhancing the net photosynthesis, which in turn alleviates the destructive effects on chloroplasts (Kovacs and Lindermayr 2013). The stimulating effect of NO on PSII photochemistry has been correlated with an increase in the proportion of the open PSII reaction centers (Shao et al. 2013) and promoting energy balance in the chloroplast (Vanlerberghe et al. 2016). Interestingly, the NO scavenger eliminated the effects of endogenous or exogenous NO on the photosynthetic performance to some extent (Chen et al. 2014a).

Many studies show that NO acts as a regulator by interacting with secondary messengers, such as $\mathrm{Ca}^{2+}$, cyclic guanosine monophosphate (cGMP), hydrogen peroxide $\left(\mathrm{H}_{2} \mathrm{O}_{2}\right)$, phytohormones such as abscisic acid (ABA), protein kinases, such as serine/threonine protein kinase (OST1), and mitogen-activated kinases (MAPK), which in turn can modulate expression of target genes that are involved in stress recovery phytohormones (Cui et al. 2011, Baudouin and Hancock 2013). Salicylic acid (SA) is

\section{Materials and methods}

Plant materials and treatments: Maize (Zea mays cv. ZhuYu 309) seeds were obtained from Zhumadian Research Institute for Agricultural Sciences. The seeds were soaked in water containing $0.2 \%(\mathrm{w} / \mathrm{v})$ tiuram and benomyl for $24 \mathrm{~h}$ to prevent fungal infection, then placed on moist gauze spread in discs, and finally germinated in the dark at $28^{\circ} \mathrm{C}$ for $4 \mathrm{~d}$. Uniformly geminated seeds were selected and transplanted to light proof plastic containers $(20 \times 15 \times 14 \mathrm{~cm}$; length $\times$ width $\times$ height $)$ of aerated halfstrength Hoagland nutrient solution with 12 seedlings per container. Seedlings were mounted on floating polyfoam board with sponges; nutrient solutions were renewed every two days and aerated once each day. All plants were grown in a light-and-temperature controlled green house with a $28 \pm 2{ }^{\circ} \mathrm{C} / 20 \pm 2^{\circ} \mathrm{C}$ day-night regime, a light intensity of $400 \mu \mathrm{mol}$ (photon) $\mathrm{m}^{-2} \mathrm{~s}^{-1}$, and a relative humidity of $80 \pm 5 \%$.

The 20-d-old plants were transferred in half-strength Hoagland nutrient solution supplemented with or without $5 \mathrm{mM}$ SA for $3 \mathrm{~d}$, and then exposed to $20 \%$ polyethylene glycol (PEG)-6000 solution (-0.8 MPa) or $0.4 \mathrm{mM} \mathrm{2-4-}$ carboxyphenyl-4,4,5,5-tetramethylimidazoline-1-oxyl-3oxide (c-PTIO) for $3 \mathrm{~d}$. The maize seedlings were treated hydroponically (Gondor et al. 2016) as follows: a well-known signaling molecule that plays multifaceted roles in the stress tolerance of plants. Many recent studies have reported the effects of exogenous NO and SA on alleviating oxidative damage induced by environment stresses, such as nickel stress on wheat (Siddiqui et al. 2013), cadmium stress on perennial ryegrass (Wang et al. 2013) and peanut ( $\mathrm{Xu}$ et al. 2015), salt stress on cotton seedlings (Liu et al. 2014) and soybean (Simaei et al. 2011) and rice (Mostofa et al. 2015). Most interestingly, $\mathrm{NO}$ might be involved in $\mathrm{H}_{2} \mathrm{O}_{2}$ - and SA-induced reduction of oxidative damage through the upregulation of antioxidant defense to confer stress tolerance (Mostofa et al. 2015).

It is reported that to adapt to WD stress, plants have evolved several photosynthetic mechanisms, such as, down-regulating the activity of enzymes related to the Calvin cycle, particularly Rubisco and Rubisco activase (RCA) involved in photochemistry (Dias et al. 2010, Xu et al. 2013). Thus, it is well known that the increase in photosynthesis can be achieved by enhancing the Rubisco and RCA activities (Carmo-Silva et al. 2013, Chen et al. $2014 b$ ). However, the role of SA in inducing photosynthetic ability in response to WD through activation of Rubisco and RCA is not clear. Moreover, the role of NO in the regulation of photosynthetic enzymes is unknown. Therefore, we used exogenous SA and NO scavenger under WD conditions, and then analyze the photosynthetic characteristics, the activities of RCA and Rubisco enzymes, and the expression of genes encoding these enzymes.

\begin{tabular}{ll}
\hline Treatment & Composition \\
\hline CK & distilled water as a control \\
WD & PEG solution for $3 \mathrm{~d}$ \\
SA + WD & SA pretreatment plus PEG \\
WD + P & PEG plus c-PTIO \\
SA + WD + P & SA pretreatment plus PEG and c-PTIO \\
\hline
\end{tabular}

Then, at the third day after treatment, the second uppermost fully expanded leaves of uniform seedlings were sampled, immediately frozen in liquid $\mathrm{N}_{2}$, and stored at $-80^{\circ} \mathrm{C}$ until (for) analysis. Four biological replicates were performed for each treatment.

Growth parameters and relative water content: Plant growth was characterized by the measurement of plant height and fresh mass in the leaves of 10 plants. The samples were placed in an oven at $105^{\circ} \mathrm{C}$ for $15 \mathrm{~min}$ and dried to a constant mass at $75^{\circ} \mathrm{C}$. The length (from ligule to leaf tip) and width (the widest portion of the leaf blade) of each green leaf were measured in situ. Individual green leaf area (LA) was estimated as the product of leaf length and maximum breadth, adjusted by a constant coefficient as follows (Elings 2000): $\mathrm{LA}=$ length $\times$ maximum width $\times 0.75$. 
The relative water content (RWC) in leaves was determined by the method described by Wu and Xia (2006). The RWC percentage was calculated using the following formula: $\mathrm{RWC}[\%]=100 \% \times[(\mathrm{FM}-\mathrm{DM}) /(\mathrm{SM}-\mathrm{DM})]$; where FM stands for fresh mass, DM for dry mass, and SM for saturated mass. SM was determined after letting the leaflet float on distilled water for $24 \mathrm{~h}$ at room temperature.

Photosynthetic rate, chlorophyll (Chl) $a$ fluorescence transients: The leaf gas-exchange parameters, such as net photosynthetic rate $\left(P_{\mathrm{N}}\right)$, stomatal conductance $\left(g_{\mathrm{s}}\right)$, and intercellular $\mathrm{CO}_{2}$ concentration $\left(C_{\mathrm{i}}\right)$ were determined using $L C A-3$ portable photosynthesis system $(A D C$, Hoddesdon, UK) between 11:00 and 13:00 $\mathrm{h}$, intrinsic water-use efficiency $\left(\mathrm{WUE}_{\mathrm{i}}=P_{\mathrm{N}} / g_{\mathrm{s}}\right)$ of each seedling was calculated. The polyphasic rise in the transient fluorescence was measured on the second fully expanded leaves from the top after 30 min of dark adaptation using a plant efficiency analyzer (PEA; Hansatech Instruments, UK), following the procedures of Strasser et al. (2000). The Chl a fluorescence transients were obtained by 2-s saturating red light and analyzed with the JIP-test (Strasser et al. 2000): the fluorescence intensity at $50 \mu \mathrm{s}$, considered $\mathrm{F}_{\mathrm{o}}$, when all PSII RCs are open; the maximal fluorescence intensity, $F_{m}$, assuming that the excitation intensity is high enough to close all of the RCs of PSII. $F_{v} / F_{m}=\left(F_{m}-F_{0}\right) / F_{m}$, represents the maximum photochemical efficiency of PSII of chloroplasts. $\mathrm{PI}_{\mathrm{ABS}}=\mathrm{RC} / \mathrm{ABS}\left[\varphi \mathrm{P}_{\mathrm{o}} /\left(1-\varphi \mathrm{P}_{\mathrm{o}}\right)\right]\left[\psi_{\mathrm{o}} /\right.$ $\left.\left(1-\psi_{\mathrm{O}}\right)\right]$, represents the photosynthesis performance in PSII electron transport.

RCA and Rubisco activity, NO concentration: Rubisco activity was measured as described by Jiang et al. (2012).
RCA activity was determined using a Rubisco Activase Assay Kit (Genmed Scientifics Inc., Wilmington, DE, USA). NO content was measured using revolutionary NO measurement system (Cheng et al. 2015) that uses microion electrodes to independently measure nitrite $\left(\mathrm{NO}_{2}^{-}\right)$and nitrate $\left(\mathrm{NO}_{3}{ }^{-}\right)$ions. The sum of these two ions gives the original amount of NO present in leaves.

Total RNA extraction and gene expression analysis: Total RNA was isolated from wheat leaves with the Spin Column Plant Total RNA Purification Kit (Sangon Biotech, Shanghai, China) according to the manufacturer's instructions. The cDNA template for real-time RT-PCR was synthesized using the AMV First Strand cDNA Synthesis Kit (Sangon Biotechnology Co., Shanghai, China). Realtime RT-PCR was performed with the ABI StepOne PlusTM Real-Time PCR Detection System (ABI, Waltham, MA, US). Each reaction $(20 \mu \mathrm{L})$ consisted of $1 \mu \mathrm{L}$ of diluted cDNA and $10 \mu \mathrm{L}$ of SybrGreen qPCR Master Mix $(A B I)$. Actin was used as an internal reference gene to calculate relative transcript levels. Relative gene expression was calculated as described by Livak and Schmittgen (2001).

Statistical analysis: Data was expressed as means \pm standard deviation (SD) of independent replicates. Based on sequences in the GenBank database, gene-specific primers (Table 1) were designed with Primer Premier 5.0 and used for amplification. Data was analyzed using oneway analysis of variance (ANOVA) by SPSS (version 19.0) and Duncan's multiple range test to determine significant $(p<0.05)$ differences between treatments.

Table 1. Primers sequences used for real-time fluorescence quantitative PCR (qRT-PCR) for detecting gene expression in the leaves of maize plants. $T_{m}-$ melting temperature.

\begin{tabular}{llll}
\hline Gene & Gene description & Sequence (5'-3') & $\mathrm{T}_{\mathrm{m}}\left[{ }^{\circ} \mathrm{C}\right]$ \\
\hline actin & & F: CTGAACCCCAAGGCAAACA & 59.0 \\
& & R: ACTGGCGTACAGGGAAAGAA & 57.3 \\
$R b c \mathrm{~L}$ & Rubisco large subunit & F: CCGTTTCGTCTTTTGTGCC & 58.9 \\
& & R: TGCGGTGAATCCTCCTGTT & 58.3 \\
$R b c \mathrm{~S}$ & Ribulose bisphosphate carboxylase & F: CGCTACTGGACCATGTGGAA & 59.0 \\
& small chain & R: ACTGCGTCTGCTTGATGTTGT & 58.1 \\
\multirow{2}{*}{$Z m R C A \alpha$} & $\alpha$-form Ribulose-1,5-bisphosphate & F: TGCAGCTTTCTCCTCCACTTC & 60.1 \\
& carboxylase/oxygenase & R: GCCATGGCTCTGACTCTGATG & 60.3 \\
\multirow{2}{*}{$Z m R C A \beta$} & $\beta$-form Ribulose-1,5-bisphosphate & F: CCGAACTAAAAAGCACAAGAAATG & 60.5 \\
& carboxylase/oxygenase & R: CAGCCATCGCCTTGAACCT & 60.3 \\
\hline
\end{tabular}

\section{Results}

Growth and RWC: Plant growth can reflect the effects of stress; thus, FW, DW, RWC, and plant height of maize were determined (Table 2). Our results showed that drought inhibited maize growth, showing 51.8, 23.2, 17.7, 4.3, and $11.9 \%$ decrease in FM, DM, RWC, plant height, and LA, respectively. However, we found that $0.5 \mathrm{mM} \mathrm{SA}$ could relieve the inhibition, and FM, DM, RWC, plant height, and LA of maize seedlings pretreated with $0.5 \mathrm{mM}$ SA followed by exposure to PEG solution were significantly higher $(14.6,2.3,8.1,17.8$, and $11.4 \%$, 
respectively) than those with WD treatment alone. The addition of c-PTIO to WD or WD+SA solution further reduced these indexes as compared with WD and WD + SA treatments, respectively.

Photosynthetic characteristics: WD strongly suppressed photosynthetic capacity. $P_{\mathrm{N}}, g_{\mathrm{s}}, \mathrm{PI}$, and $\mathrm{WUE}_{\mathrm{i}}$ of leaves significantly decreased by $53.2,39.6,28.8,25.0 \%$, respectively, as compared with $\mathrm{CK}$ treatment (Table 3), but $C_{\mathrm{i}}$ increased by $41.4 \%$. However, WD did not have any effect on $\mathrm{F}_{\mathrm{v}} / \mathrm{F}_{\mathrm{m}}$ as compared to control. SA pretreatment under WD enhanced the $g_{\mathrm{s}}, P_{\mathrm{N}}, \mathrm{F}_{\mathrm{v}} / \mathrm{F}_{\mathrm{m}}, \mathrm{PI}$, and $\mathrm{WUE}_{\mathrm{i}}$ by $33.6,48.0,6.8,18.1$, and $18.0 \%$, respectively, as compared with WD treatment. As an NO scavenger, the addition of c-PTIO to WD or SA solution could decrease these parameters as compared with WD or WD + SA treatments, respectively.

Table 2. Effect of salicylic acid (SA) and 2-4-carboxyphenyl-4,4,5,5-tetramethylimidazoline-1-oxyl-3-oxide (c-PTIO) on fresh mass (FM), dry mass (DM), relative water content (RWC), plant height, and leaf area of maize seedlings exposed to $20 \%$ PEG-stimulated water deficit. Data are the means \pm SD of four replicates. Different letters in the same columns showed significant differences at $p<0.05$. The 20-d-old plants were pretreated with $5 \mathrm{mM} \mathrm{SA}$ for $3 \mathrm{~d}$, then exposed to PEG solution or c-PTIO for $3 \mathrm{~d}$. The maize seedlings were treated as follows: CK - distilled water as a control; WD - 20\% PEG-6000 solution for $3 \mathrm{~d}$; SA + WD -5 mM SA pretreatment plus 20\% PEG-6000; WD + P - 20\% PEG-6000 + 0.4 mM c-PTIO; SA + WD + P - 5 mM SA pretreatment + 20\% PEG-6000 and 0.4 mM carboxy-PTIO.

\begin{tabular}{llllll}
\hline Treatment & FM $[\mathrm{g}]$ & DM $[\mathrm{g}]$ & RWC $[\%]$ & Plant height $[\mathrm{cm}]$ & Leaf area $\left[\mathrm{cm}^{2}\right]$ \\
\hline CK & $3.40 \pm 0.27^{\mathrm{a}}$ & $0.56 \pm 0.02^{\mathrm{a}}$ & $90.03 \pm 5.03^{\mathrm{a}}$ & $25.24 \pm 1.31^{\mathrm{a}}$ & $140.29 \pm 3.10^{\mathrm{a}}$ \\
WD & $1.64 \pm 0.05^{\mathrm{c}}$ & $0.43 \pm 0.01^{\mathrm{bc}}$ & $74.05 \pm 4.10^{\mathrm{c}}$ & $18.97 \pm 0.73^{\mathrm{c}}$ & $123.56 \pm 5.01^{\mathrm{b}}$ \\
SA + WD & $1.88 \pm 0.10^{\mathrm{b}}$ & $0.44 \pm 0.00^{\mathrm{b}}$ & $80.05 \pm 2.35^{\mathrm{b}}$ & $22.34 \pm 1.02^{\mathrm{b}}$ & $137.67 \pm 10.34^{\mathrm{ab}}$ \\
WD + P & $1.27 \pm 0.12^{\mathrm{d}}$ & $0.40 \pm 0.01^{\mathrm{c}}$ & $68.04 \pm 2.09^{\mathrm{d}}$ & $17.92 \pm 0.62^{\mathrm{c}}$ & $110.74 \pm 7.81^{\mathrm{c}}$ \\
SA + WD + P & $1.43 \pm 0.12^{\mathrm{d}}$ & $0.42 \pm 0.01^{\mathrm{bc}}$ & $76.04 \pm 3.03^{\mathrm{c}}$ & $20.38 \pm 1.02^{\mathrm{bc}}$ & $125.65 \pm 10.67^{\mathrm{b}}$ \\
\hline
\end{tabular}

Table 3. Effects of salicylic acid (SA) and 2-4-carboxyphenyl-4,4,5,5-tetramethylimidazoline-1-oxyl-3-oxide (c-PTIO) on photosynthetic characteristics of maize seedlings exposed to $20 \%$ PEG-stimulated water deficit. Data are the means \pm SD of four replicates. Different letters in the same columns showed significant differences at $p<0.05$. The 20 -d-old plants were pretreated with 5 $\mathrm{mM}$ SA for $3 \mathrm{~d}$, and then exposed to PEG solution or c-PTIO for $3 \mathrm{~d}$. The maize seedlings were treated as follows: CK - distilled water as a control; WD - 20\% PEG-6000 solution for $3 \mathrm{~d}$; SA + WD - 5 mM SA pretreatment plus 20\% PEG-6000; WD + P - 20\% PEG$6000+0.4$ mM c-PTIO; SA + WD + P - 5 mM SA pretreatment + 20\% PEG-6000 and 0.4 mM carboxy-PTIO.

\begin{tabular}{lllllll}
\hline Treatment & $\begin{array}{l}g_{\mathrm{s}} \\
{\left[\mathrm{mmol}\left(\mathrm{H}_{2} \mathrm{O}\right) \mathrm{m}^{-2} \mathrm{~s}^{-1}\right]}\end{array}$ & $\begin{array}{l}C_{\mathrm{i}} \\
{\left[\mu \operatorname{mol}\left(\mathrm{CO}_{2}\right) \mathrm{mol}^{-1}\right]}\end{array}$ & $\begin{array}{l}P_{\mathrm{N}} \\
{\left[\mu \operatorname{mol}\left(\mathrm{CO}_{2}\right) \mathrm{m}^{-2} \mathrm{~s}^{-1}\right]}\end{array}$ & $\mathrm{F}_{\mathrm{v}} / \mathrm{F}_{\mathrm{m}}$ & PI & $\begin{array}{l}\mathrm{WUE}_{\mathrm{i}} \\
{\left[\mu \mathrm{mol}\left(\mathrm{CO}_{2}\right) \mathrm{mmol}\left(\mathrm{H}_{2} \mathrm{O}\right)^{-1}\right]}\end{array}$ \\
\hline $\mathrm{CK}$ & $350.31 \pm 5.00^{\mathrm{a}}$ & $105.71 \pm 10.23^{\mathrm{c}}$ & $9.58 \pm 0.53^{\mathrm{a}}$ & $0.79 \pm 0.00^{\mathrm{a}}$ & $1.63 \pm 0.12^{\mathrm{a}}$ & $2.52 \pm 0.31^{\mathrm{a}}$ \\
$\mathrm{WD}$ & $211.56 \pm 24.13^{\mathrm{c}}$ & $149.52 \pm 4.72^{\mathrm{a}}$ & $4.48 \pm 0.41^{\mathrm{c}}$ & $0.73 \pm 0.06^{\mathrm{ab}}$ & $1.16 \pm 0.36^{\mathrm{b}}$ & $1.89 \pm 0.13^{\mathrm{b}}$ \\
$\mathrm{SA}+\mathrm{WD}$ & $282.55 \pm 13.56^{\mathrm{b}}$ & $126.78 \pm 5.36^{\mathrm{b}}$ & $6.63 \pm 0.40^{\mathrm{b}}$ & $0.78 \pm 0.02^{\mathrm{a}}$ & $1.37 \pm 0.42^{\mathrm{a}}$ & $2.23 \pm 0.12^{\mathrm{a}}$ \\
$\mathrm{WD}+\mathrm{P}$ & $180.01 \pm 2.89^{\mathrm{d}}$ & $150.01 \pm 9.54^{\mathrm{a}}$ & $4.08 \pm 0.20^{\mathrm{d}}$ & $0.67 \pm 0.04^{\mathrm{b}}$ & $0.77 \pm 0.29^{\mathrm{c}}$ & $1.69 \pm 0.02^{\mathrm{c}}$ \\
$\mathrm{SA}+\mathrm{WD}+\mathrm{P}$ & $188.94 \pm 1.77^{\mathrm{cd}}$ & $148.33 \pm 8.54^{\mathrm{a}}$ & $5.02 \pm 0.56^{\mathrm{c}}$ & $0.75 \pm 0.04^{\mathrm{a}}$ & $1.66 \pm 0.28^{\mathrm{a}}$ & $2.04 \pm 0.02^{\mathrm{b}}$ \\
\hline
\end{tabular}

Activities of Rubisco and RCA enzymes, and NO content: We determined Rubisco and RCA activities to study further the mechanism by which SA regulates $\mathrm{CO}_{2}$ fixation. Rubisco and RCA activity were respectively decreased by 45.3 and $39.9 \%$ in response to WD (Fig. 1). However, SA-pretreated maize plants had higher Rubisco and RCA activities than plants without SA treatment under WD. We found that as compared with WD and WD + SA treatments, the Rubisco activity was decreased by 15.5 and $17.9 \%$ with WD + c-PTIO and WD + SA + c-PTIO treatments, respectively. Also, RCA activity was reduced by 40.0 and $36.6 \%$ in WD + SA + c-PTIO treatments, respectively. In addition, determination of NO content showed that WD increased NO production, especially SA + WD treatment increased NO con tent by 4.1 fold and
1.2 fold compared with CK and WD.

Expression of $\boldsymbol{R} \boldsymbol{b} \boldsymbol{c}$ and $\boldsymbol{R} \boldsymbol{C A}$ genes: To evaluate further the molecular response of maize photosynthesis to SA and c-PTIO under WD, we analyzed the genes encoding the $R C A$ and $R b c$ subunits at the transcriptional level using qRT-PCR (Fig. 2). Although under WD the $Z m R C A \alpha$, $Z m R C A \beta, R b c \mathrm{~L}$ and $R b c \mathrm{~S}$ levels were decreased by 57.4, $87.4,37.9$, and $59.3 \%$, respectively, they were significantly down-regulated except $R b c \mathrm{~S}$ when maize plants were treated with c-PTIO. However, transcript levels of $Z m R C A \alpha, Z m R C A \beta$ and $R b c \mathrm{~L}$ in SA + WD treatment were higher than those in WD treatment but decreased due to the addition of c-PTIO. 


\section{Discussion}

WD is one of most damaging of environmental stresses for plant growth, development, and yield formation. Plant responses to WD stress are complicated and involve processes regulated by signaling molecules such as $\mathrm{NO}$ and SA (Iqbal et al. 2014, Qiao et al. 2014). Our results showed that WD resulted in the loss of leaf water content and inhibition of plant growth; however, SA pretreatment significantly relieved WD-induced inhibition of growth and resulted in increased FW, DW, LA, and plant height. Interestingly, these stimulating effects were suppressed by the NO scavenger c-PTIO. This indicated that NO signal might be involved in the adaptation of SA-regulated maize seedling growth to WD.

Photosynthesis, the main force driving crop production, is one of the key processes to be affected by WD (Ashraf et al. 2013). Photosynthetic $\mathrm{CO}_{2}$ assimilation per unit area and time, i.e., $P_{\mathrm{N}}$ is inhibited by rapidly developing $\mathrm{WD}$ in physiological studies, so it is implicated in a decrease in dry matter production (Tatar et al. 2016). In this study, substantial decrease of photosynthesis under WD condition was due to nonstomatal factors, the photosynthetic capacity was impaired significantly, because the chloroplast envelope was possibly affected by low RWC $(<80 \%)$. However, SA pretreatment could reverse such changes. Moreover, SA enhanced $P_{\mathrm{N}}$ by maintaining hydraulic factors (RWC), which serves to regulate water loss in relation to water uptake. Therefore, RWC and $\mathrm{WUE}_{\mathrm{i}}$ in $\mathrm{WD}+\mathrm{SA}$ pretreatment decreased very little compared with control. NO signal is produced in the chloroplast, and is thought to regulate photosynthesis relying on nonstomatal factors under stresses (Kausar and Shahbaz 2013, Procházková et al. 2013). NO was also involved in CTK regulation of photosynthetic adaptability to WD in our previous experiment (Shao et al. 2010). Our study suggests that SA-induced improvements in $\mathrm{CO}_{2}$ assimilation may also be related to NO signals. Therefore, NO scavenger may manifest changes in $\mathrm{CO}_{2}$ fixation rate and electron transport ability.

The activation of Rubisco by RCA serves an important regulatory function in linking the rate of $\mathrm{CO}_{2}$ fixation to the rate of electron transport activity via the production of ATP (Dias et al. 2010, Parry et al. 2013). Rubisco catalyzes the first step in the net photosynthetic $\mathrm{CO}_{2}$ assimilation (Chen et al. 2014b), and its activation state is regulated by RCA (Boex-Fontvieille et al. 2014). In this study, water loss caused de-activation of Rubisco and RCA, which in turn caused a metabolic limitation to photosynthesis, resulting in a drop of net formation of carbohydrates, such as dry mass. However, there was no significant difference in Rubisco and RCA activity between SA + WD and WD treatments regarding an increase in $\mathrm{CO}_{2}$ assimilation rate. These results suggest that SA improves the efficiency of photosynthetic carbon fixation by activating the photosynthetic enzymes, which restore the supply of $\mathrm{CO}_{2}$ to Rubisco enzyme and help in overcoming stomatal limitations.

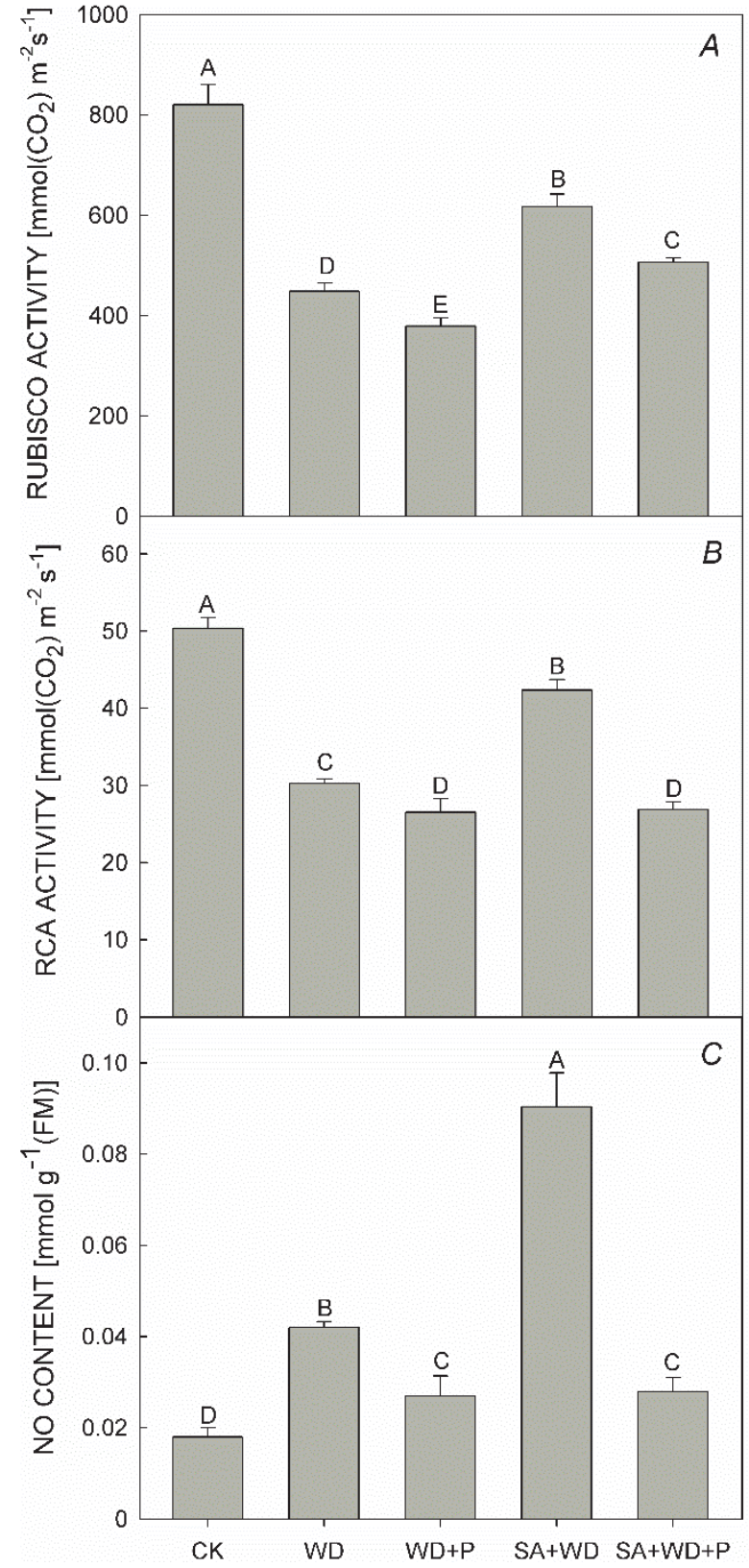

Fig. 1. Effects of salicylic acid (SA) and 2-4-carboxyphenyl4,4,5,5-tetramethylimidazoline-1-oxyl-3-oxide (c-PTIO) on Rubisco activity $(A)$, Rubisco activase activity $(B)$, and nitric oxid (NO) content $(C)$ of maize seedlings under $20 \%$ polyethylene glycol (PEG)-6000 induced water-deficit stress. Data are the means \pm SD of three replicates. Different letters in the same columns showed significant differences at $p<0.05$. The 20-d-old plants were pretreated hydroponically with $5 \mathrm{mM} \mathrm{SA}$ solution for $3 \mathrm{~d}$, and then exposed to $20 \%$ PEG solution or $0.4 \mathrm{mM}$ c-PTIO for $3 \mathrm{~d}$. The maize seedlings were treated as follows: CK - distilled water as a control; WD - 20\% PEG-6000 solution for $3 \mathrm{~d}$; SA + WD - $5 \mathrm{mM}$ SA pretreatment plus $20 \%$ PEG-6000; WD + P - 20\% PEG-6000 + 0.4 mM c-PTIO; SA + $\mathrm{WD}+\mathrm{P}-5 \mathrm{mM}$ SA pretreatment $+20 \%$ PEG-6000 and $0.4 \mathrm{mM}$ c-PTIO. 


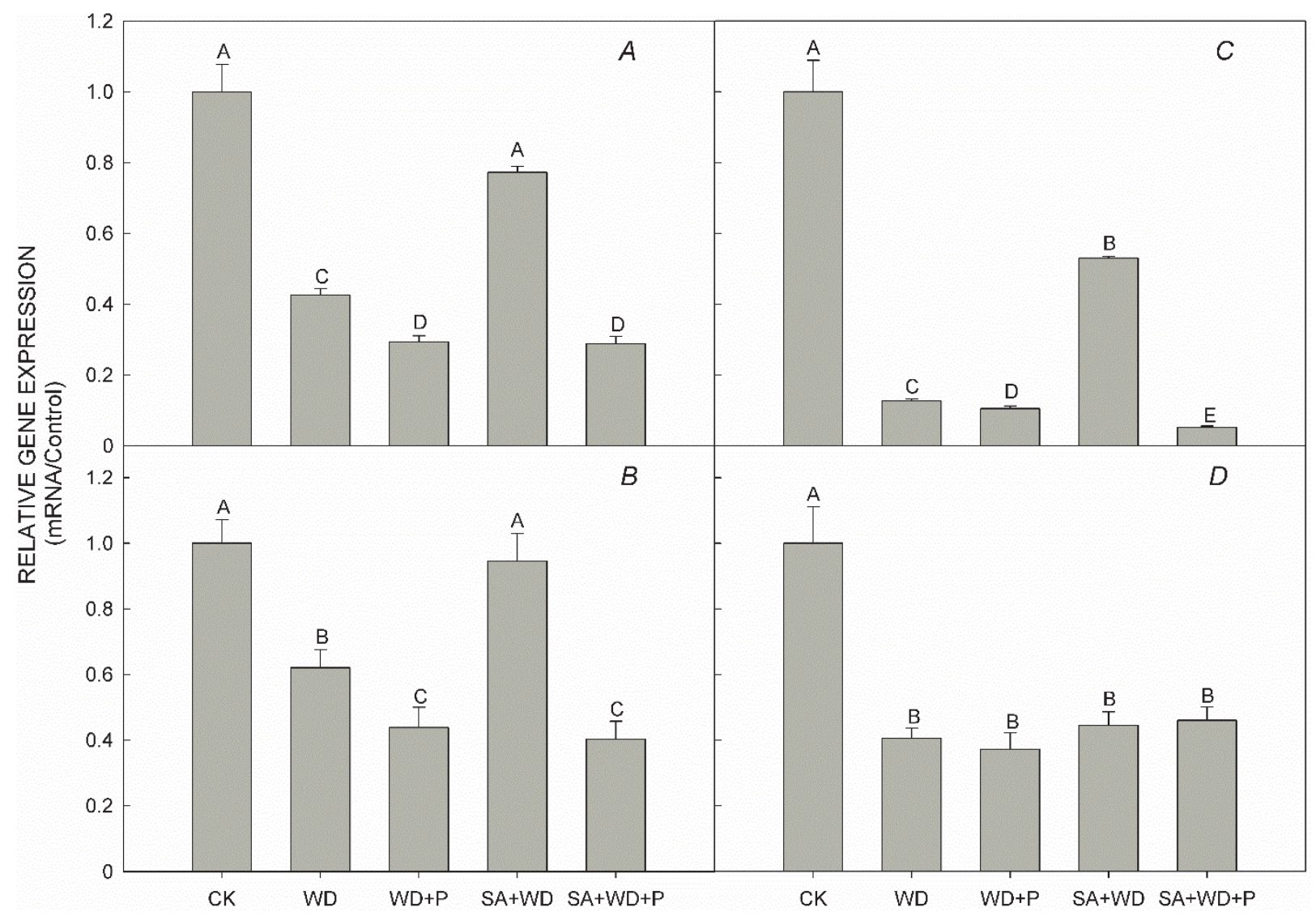

Fig. 2. Effects of salicylic acid (SA) and 2-4-carboxyphenyl-4,4,5,5-tetramethylimidazoline-1-oxyl-3-oxide (c-PTIO) on the expression of $Z m R C A \alpha(A), R b c \mathrm{~L}(B), Z m R C A \beta(C)$, and $R b c \mathrm{~S}(D)$ of maize seedlings under $20 \%$ polyethylene glycol (PEG)- 6000 induced waterdeficit stress. Data are the means \pm SD of three replicates. Different letters in the same columns showed significant differences at $p<0.05$. The 20-d-old plants were pretreated hydroponically with $5 \mathrm{mM} \mathrm{SA}$ solution for $3 \mathrm{~d}$, then exposed to $20 \%$ PEG solution or $0.4 \mathrm{mM}$ c-PTIO for $3 \mathrm{~d}$. The maize seedlings were treated as follows: CK - distilled water as a control; WD - 20\% PEG-6000 solution for $3 \mathrm{~d}$; $\mathrm{SA}+\mathrm{WD}-5 \mathrm{mM}$ SA pretreatment plus 20\% PEG-6000; WD + P - 20\% PEG-6000 + $0.4 \mathrm{mM} \mathrm{c-PTIO}$; SA + WD + P - 5 mM SA pretreatment $+20 \%$ PEG-6000 and $0.4 \mathrm{mM}$ c-PTIO.

The amount of Rubisco synthesized is primarily determined by the levels of Rubisco subunits $(R b c \mathrm{~L}, R b c \mathrm{~S})$ mRNA (Suzuki and Makino 2013). In the present study, $R b c \mathrm{~S}$ showed some upregulation, but $R b c \mathrm{~L}$ was significantly up-regulated by SA, suggesting that SA could enhance Rubisco biosynthesis under WD. Two RCA genes, $Z m R C A \alpha$ and $Z m R C A \beta$, encoding larger and smaller polypeptides of approximately 46 and $43 \mathrm{kD}$ were found in maize (Yin et al. 2014), which ensure a stable RCA structure and maintain initial Rubisco activity under stress conditions (Wang et al. 2010, Chen et al. 2014b). Different RCA subunits may play different roles in photosynthetic acclimation. Under drought, RCA $\beta$ subunit was reported to decrease significantly in wheat (Zhao et al. 2016). In the present study, although $Z m R C A \alpha$ and $Z m R C A \beta$ genes expression were down-regulated under $\mathrm{WD}$, a significant increase in these genes (consistent with an increase in Rubisco activity) was observed after SA pretreatment. Therefore, we speculated that enhanced transcription of $Z m R C A \alpha$ and $Z m R C A \beta$ mRNA under WD caused an increase in the initial Rubisco activity after SA treatment. However, c-PTIO lowered the transcription of $Z m R C A \alpha$ and $Z m R C A \beta$ mRNA and was accompanied by the down-regulation of $P_{\mathrm{N}}$ and photosynthetic performance implying that $\mathrm{SA}$ improving photosynthetic adaptability might be related to the NO signal. Moreover, our results show that NO content under WD conditions were significantly different from those in SA or c-PTIO treatments, which further implicated NO in SA-induced photosynthetic adaptability.

In conclusion, WD significantly decreased photosynthesis characteristics due to nonstomatal limitations, such as leaf $\mathrm{CO}_{2}$ assimilation rate, PSII activity, the amount, and activity of Rubisco and RCA enzymes. However, SA exerts a positive effect on photosynthesis under WD condition, which occurs mainly through stimulating the transcription of $R b c \mathrm{~L}, Z m R C A \alpha$, and $Z m R C A \beta$ mRNA and enhancing the activity of Rubisco and RCA. Moreover, the NO scavenger highlights an important role for NO in SAregulated leaf photosynthesis adaptability to WD stress. 


\section{References}

Ashraf M., Harris P.J.C.: Photosynthesis under stressful environments: an overview. - Photosynthetica 51: 163-190, 2013.

Bajguz A.: Nitric oxide: role in plants under abiotic stress. - In: Parvaiz A., Mohd R.W. (ed.): Biomedical and Life Sciences: Physiological Mechanisms and Adaptation Strategies in Plants under Changing Environment. Pp. 137-159. Springer, New York 2013.

Boex-Fontvieille E., Daventure M., Jossier M. et al.: Phosphorylation pattern of Rubisco activase in Arabidopsis leaves. Plant Biol. 16: 550-557, 2014.

Carmo-Silva A.E., Salvucci M.E.: The regulatory properties of rubisco activase differ among species and affect photosynthetic induction during light transitions. - Plant Physiol. 161: 16451655,2013

Chen P., Li X., Huo K. et al.: Promotion of photosynthesis in transgenic rice over-expressing of maize $\mathrm{C} 4$ phosphoenolpyruvate carboxylase gene by nitric oxide donors. - J. Plant Physiol. 171: 458-466, 2014a.

Chen Y., Jin J.H., Jiang Q.S. et al.: Sodium bisulfite enhances photosynthesis in rice by inducing Rubisco activase gene expression. - Photosynthetica 52: 475-478, 2014b.

Cheng T., Chen J., Ef A.A. et al.: Quantitative proteomics analysis reveals that S-nitrosoglutathione reductase (GSNOR) and nitric oxide signaling enhance poplar defense against chilling stress. - Planta 242: 1361-1390, 2015.

Corpas F.J., Leterrier M., Valderrama R. et al.: Nitric oxide imbalance provokes a nitrosative response in plants under abiotic stress. - Plant Sci. 181: 604-611, 2011.

Cui J.X., Zhou Y.H., Ding J.G. et al.: Role of nitric oxide in hydrogen peroxide-dependent induction of abiotic stress tolerance by brassinosteroids in cucumberpce. - Plant Cell Environ. 34: 347-358, 2011.

Dias M.C., Brüggemann W.: Limitations of photosynthesis in phaseolus vulgaris under drought stress: gas exchange, chlorophyll fluorescence and Calvin cycle enzymes. - Photosynthetica 48: 96-102, 2010.

Elings A.: Estimation of leaf area in tropical maize. - Agron. J. 92: 436-444, 2000.

FAOSTAT: Food and agricultural commodities production. http://faostat.fao.org/site/339/default.aspx , 2010.

Gondor O. K., Janda T., Soós V. et al.: Salicylic acid induction of flavonoid biosynthesis pathways in wheat varies by treatment. - Front. Plant Sci. 7: 1447, 2016.

Hancock J.T.: NO synthase? Generation of nitric oxide in plants. - Period. Biol. 114: 19-24, 2012.

Iqbal N., Umar S., Khan N.A. et al.: A new perspective of phytohormones in salinity tolerance: Regulation of proline metabolism. - Environ. Exp. Bot. 100: 34-42, 2014.

Jasid S., Simontacchi M., Bartoli C.G. et al:: Chloroplasts as a nitric oxide cellular source. Effect of reactive nitrogen species on chloroplastic lipids and proteins. - Plant Physiol. 142: 1246$1255,2006$.

Jiang Y.P., Cheng F., Zhou Y.H. et al.: Cellular glutathione redox homeostasis plays an important role in the brassinosteroidinduced increase in $\mathrm{CO}_{2}$ assimilation in Cucumis sativus. New Phytol. 194: 932-943, 2012.

Kausar F., Shahbaz M.: Interactive effect of foliar application of nitric oxide (NO) and salinity on wheat (Triticum aestivum L.). - Pak. J. Bot. 45: 67-73, 2013.

Kovacs I., Lindermayr C.: Nitric oxide-based protein modification: formation and site-specificity of protein S-nitrosylation.
- Front. Plant Sci. 4: 137, 2013.

Liao W.B., Huang G.B., Yu J.H. et al.: Nitric oxide and hydrogen peroxide alleviate drought stress in marigold explants and promote its adventitious root development. - Plant Physiol. Bioch. 58: 6-15, 2012.

Livak K.J., Schmittgen T.D.: Analysis of relative gene expression data using real-time quantitative PCR and the $2-$ $\Delta \Delta \mathrm{CT}$ method. - Methods 25: 402-408, 2001.

Liu S., Dong Y., Xu L. et al.: Effects of foliar applications of nitric oxide and salicylic acid on salt-induced changes in photosynthesis and antioxidative metabolism of cotton seedlings. - Plant Growth Regul. 73: 67-78, 2014.

Mostofa M.G., Fujita M., Tran L.S.P.: Nitric oxide mediates hydrogen peroxide-and salicylic acid-induced salt tolerance in rice (Oryza sativa L.) seedlings. - Plant Growth Regul. 77: 265-277, 2015.

Parry M.A.J., Andralojc P.J., Scales J.C.: Rubisco activity and regulation as targets for crop improvement. - J. Exp. Bot. 64: 717-730, 2013.

Procházková D., Haisel D., Wilhelmová N. et al.: Effects of exogenous nitric oxide on photosynthesis. - Photosynthetica 51: 483-489, 2013

Qiao W., Li C., Fan L.M.: Cross-talk between nitric oxide and hydrogen peroxide in plant responses to abiotic stresses. Environ. Exp. Bot. 100: 84-93, 2014.

Serraj R., McNally K.L., Slamet-Loedin I. et al.: Drought resistance improvement in rice: an integrated genetic and resource management strategy. - Plant Prod. Sci. 14: 1-14, 2011.

Shao R., Wang K., Shangguan Z.P.: Cytokinin-induced photosynthetic adaptability of Zea mays L. to drought stress associated with nitric oxide signal: probed by ESR spectroscopy and fast OJIP fluorescence rise. - J. Plant Physiol. 167: 472-479, 2010

Shao R.X., Chen J.H., Miao F. et al.: Photosynthetic performance of Triticum aestivum L. in response to water and nitrogen deficit. - J. Food Agric. Environ. 11: 1252-1256, 2013.

Siddiqui M.H., Al-Whaibi M.H., Ali H.M. et al.: Mitigation of nickel stress by the exogenous application of salicylic acid and nitric oxide in wheat. - Aust. J. Crop Sci. 7: 1780-1788, 2013.

Sikder S., Foulkes J., West H. et al.: Evaluation of photosynthetic potential of wheat genotypes under drought condition. Photosynthetica 53: 47-54, 2015.

Simaei M., Khavarinejad R.A., Saadatmand S. et al.: Interactive effects of salicylic acid and nitric oxide on soybean plants under NaCl salinity. - Russ. J. Plant Physl+ 58: 783-790, 2011.

Strasser R.J., Srivastava A., Tsimilli-Michael M.: The fluorescence transient as a tool to characterize and screen photosynthetic samples. - In: Yunus M., Pathre U., Mohanty P. (ed.): Probing Photosynthesis: Mechanisms, Regulation and Adaptation. Pp. 445-483. Taylor and Francis, London 2000.

Suzuki Y., Makino A.: Translational downregulation of RBCL is operative in the coordinated expression of Rubisco genes in senescent leaves in rice. - J. Exp. Bot. 64: 1145-1152, 2013.

Tatar O., Brück H., Asch F.: Photosynthesis and remobilization of dry matter in wheat as affected by progressive drought stress at stem elongation stage. - J. Agro. Crop Sci. 202: 292-299, 2016.

Tossi V., Cassia R., Bruzzone S. et al.: ABA says NO to UV-B: a universal response? - Trends Plant Sci. 17: 510-517, 2012.

Vanlerberghe G.C., Martyn G.D., Dahal K.: Alternative oxidase: 
a respiratory electron transport chain pathway essential for maintaining photosynthetic performance during drought stress. - Physiol. Plantarum 157: 322-337, 2016.

Wang G.P., Hui Z., Li F. et al.: Improvement of heat and drought photosynthetic tolerance in wheat by over accumulation of glycinebetaine. - Plant Biotechnol. Rep. 4: 213-222, 2010.

Wang Y., Suo B., Zhao T. et al.: Effect of nitric oxide treatment on antioxidant responses and $p s b A$ gene expression in two wheat cultivars during grain filling stage under drought stress and rewatering. - Acta Physiol. Plant. 33: 1923, 2011.

Wang Q., Liang X., Dong Y. et al.: Effects of exogenous salicylic acid and nitric oxide on physiological characteristics of perennial ryegrass under cadmium stress. - J. Plant Growth Regul. 32: 721-731, 2013.

Wu Q.S., Xia R.X.: Arbuscular mycorrhizal fungi influence growth, osmotic adjustment and photosynthesis of citrus under well-watered and water stress conditions. - J. Plant Physiol. 163: 417-425, 2006.
Xu L., Yu J., Han L. et al.: Photosynthetic enzyme activities and gene expression associated with drought tolerance and postdrought recovery in Kentucky bluegrass. - Environ. Exp. Bot. 89: 28-35, 2013.

Xu L.L., Fan Z.Y., Dong Y.J. et al.: Effects of exogenous salicylic acid and nitric oxide on physiological characteristics of two peanut cultivars under cadmium stress. - Biol. Plantarum 59: 171-182, 2015

Yin Z.T., Zhang Z.L., Deng D.X. et al.: Characterization of rubisco activase genes in maize: an $\alpha$-isoform gene functions alongside a $\beta$-isoform gene. - Plant Physiol. 164: 2096-2106, 2014.

Zhao G.W., Xu H.L., Zhang P.J. et al.: Effects of 2,4-epibrassinolide on photosynthesis and Rubisco activase gene expression in Triticum aestivum L. seedlings under a combination of drought and heat stress. - Plant Growth Regul. 81: 377-384, 2017. 